\title{
Erratum to: Ethnic Differences in Persistence with COPD Medications: a Register-Based Study
}

\author{
Yusun $\mathrm{Hu}^{1}$ • Lourdes Cantarero-Arévalo ${ }^{1}$ - Anne Frølich ${ }^{2}$ • Ramune Jacobsen ${ }^{2,3}$
}

Published online: 9 May 2017

(C) W. Montague Cobb-NMA Health Institute 2017

\section{ERRATUM TO: J. Racial and Ethnic Health Disparities DOI 10.1007/s40615-017-0359-8}

In Table 3, the second-column heading should be changed to "Combination therapy" and the third-column heading "Multiple drug therapy" as illustrated in this correct version.
The online version of the original article can be found at http://dx.doi.org/ 10.1007/s40615-017-0359-8

Ramune Jacobsen

ramune.jacobsen@regionh.dk

1 Faculty of Health and Medical Sciences, Department of Pharmacy, Section for Social and Clinical Pharmacy, University of Copenhagen, Universitetsparken 2, Copenhagen, Denmark

2 Research Unit for Chronic Conditions, Department of Clinical Epidemiology, Copenhagen University Hospital, Bispebjerg and Frederiksberg, Bispebjerg Bakke 23, 2400 Copenhagen, Denmark

3 Research Unit for Dietary Studies, The Parker Institute, Copenhagen University Hospital, Bispebjerg and Frederiksberg, Nordre Fasanvej, 57 Frederiksberg, Denmark
Table 3 Baseline characteristics of COPD patients using COPD maintenance therapy

\begin{tabular}{|c|c|c|c|c|}
\hline & Monotherapy & $\begin{array}{l}\text { Combination } \\
\text { therapy }\end{array}$ & $\begin{array}{l}\text { Multiple } \\
\text { drug therapy }\end{array}$ & Total \\
\hline Patients $(\mathrm{N}, \%)$ & $306(27.1)$ & $642(56.9)$ & $181(16.0)$ & 1129 \\
\hline \multicolumn{5}{|l|}{ Age } \\
\hline $36-50$ years old, $\%$ & 6.5 & 11.7 & 9.4 & 9.9 \\
\hline $51-65$ years old, $\%$ & 33.3 & 35.5 & 29.3 & 33.9 \\
\hline $66-75$ years old, $\%$ & 33.7 & 32.9 & 39.8 & 34.2 \\
\hline$>75$ years old, $\%$ & 26.5 & 19.9 & 21.6 & 22.0 \\
\hline \multicolumn{5}{|l|}{ Sex } \\
\hline Males, $\%$ & 40.2 & 42.4 & 47.0 & 42.5 \\
\hline Females, $\%$ & 59.8 & 57.6 & 53.0 & 57.5 \\
\hline \multicolumn{5}{|l|}{ Ethnicity } \\
\hline Danes, $\%$ & 94.1 & 92.4 & 95.0 & 93.3 \\
\hline Ethnic minorities, \% & 5.9 & 7.6 & 5.0 & 6.7 \\
\hline \multicolumn{5}{|c|}{ Education } \\
\hline $\begin{array}{l}\text { Higher education } \\
\text { (>10 year), \% }\end{array}$ & 38.6 & 39.6 & 39.8 & 39.3 \\
\hline $\begin{array}{l}\text { Other education } \\
\text { (<10 years), } \%\end{array}$ & 61.4 & 60.4 & 60.2 & 60.7 \\
\hline \multicolumn{5}{|l|}{ Income } \\
\hline $\begin{array}{l}\text { Low (1st } \\
\text { quartile), \% }\end{array}$ & 25.2 & 26.8 & 22.7 & 25.7 \\
\hline $\begin{array}{l}\text { Medium (2nd-3rd } \\
\text { quartiles), } \%\end{array}$ & 47.1 & 49.9 & 49.2 & 49.0 \\
\hline $\begin{array}{l}\text { High (4th } \\
\quad \text { quartile), \% }\end{array}$ & 27.8 & 23.4 & 28.2 & 25.3 \\
\hline \multicolumn{5}{|l|}{ Employment } \\
\hline Employed/retired \% & 78.1 & 71.8 & 78.5 & 74.6 \\
\hline Early retired, \% & 13.7 & 18.4 & 13.3 & 16.3 \\
\hline Unemployed, \% & 8.2 & 9.8 & 8.3 & 9.1 \\
\hline \multicolumn{5}{|l|}{ Disease severity } \\
\hline More severe, $\%$ & 18.3 & 19.9 & 22.1 & 19.8 \\
\hline Less severe, \% & 81.7 & 80.1 & 77.9 & 80.2 \\
\hline
\end{tabular}

